ARTíCULO

\title{
Patrones históricos y escenarios térmicos futuros en mares mexicanos
}

\author{
Historical patterns and predicted thermal scenarios in Mexican seas
}

\author{
Romeo Saldívar-Lucio ${ }^{1}$, Christian Salvadeo ${ }^{2}$, Pablo Del Monte-Luna ${ }^{1}$, Francisco \\ Arreguín-Sánchez ${ }^{1}$, Héctor Villalobos ${ }^{1}$, Daniel Lluch-Belda $\dagger^{1}$, Germán \\ Ponce-Díaz ${ }^{1}$, José Luis Castro-Ortiz ${ }^{1}$, José Alberto Zepeda-Domínguez ${ }^{\mathbf{1}}$, \\ Fernando Aranceta-Garza ${ }^{1}$ y Luis César Almendarez-Hernández ${ }^{1}$
}

\begin{abstract}
${ }^{1}$ Centro Interdisciplinario de Ciencias Marinas, Instituto Politécnico Nacional, Ave. Instituto Politécnico Nacional s/n, Col. Playa Palo de Santa Rita, Apartado Postal 592, CP 23096, La Paz, B.C.S., México. romeo26_1979@yahoo.com ${ }^{2}$ Universidad Autónoma de Baja California Sur, Carretera al Sur Km. 5.5, Apartado postal 19-B, C.P. 23080, La Paz, Baja California Sur, México

Abstract.- Into different areas (e.g., academic, public) predictions of climate as part of the process of decision-making are required. Despite such information need, the inconsistency of global models to predict the state of the climate in small scales (regions) is widely recognized. Considering this, we tested predictions of sea surface temperature (SST) in 10 marine regions off the coast of Mexico. Using classification and regression trees, Mexican coastal states were grouped accordingly to their similarity in instrumental records of air temperature (AST). Such AST groups were considered explanatory variables together with regional climatic scale indices (e.g., Pacific Decadal Oscillation, PDO). Historical patterns of change (period, amplitude and phase) of AST and climate indices were characterized, and then its relationship with SST was analyzed using generalized additive models (GAM). The SST response to climatic scenarios was evaluated with 3 different forcing criteria. The GAM models showed significant fits and relatively high values of $R^{2}$ and deviance. Projections of regional climate variability showed substantial differences in comparison to the monotonic increase in SST global models outputs. The rescaling strategy applied in this work for Mexican seas surface temperature, proved to be useful to integrate the historical variation with different forcing criteria.
\end{abstract}

Key words: Climate change scenarios, historical climate patterns, climate forecasting, ocean climate, regional climate representativeness

\begin{abstract}
Resumen.- En diversos ámbitos (e.g., académico, público) se requieren predicciones del clima como parte del proceso de toma de decisiones. A pesar de la citada necesidad de información por parte de sectores productivos y servidores públicos, es reconocida la inconsistencia de los modelos globales al predecir el estado del clima en escalas menores (regiones). Considerando lo anterior, se ensayaron las predicciones de la temperatura superficial del mar (TSM) en 10 regiones marinas frente a las costas de México. Mediante árboles de regresión y clasificación se agrupó a los estados costeros según su parecido en registros instrumentales de temperatura del aire (TSA). Tales grupos de TSA se tomaron como variables explicativas regionales junto con índices climáticos de mayor escala (e.g., Oscilación Decadal del Pacífico, ODP). Se caracterizaron los patrones históricos de cambio (Periodo, amplitud y fase) de TSA e índices climáticos y se analizó su relación con la TSM mediante modelos aditivos generalizados (GAM). La respuesta de la TSM ante escenarios climáticos fue evaluada bajo 3 criterios de forzamiento diferentes. Los modelos GAM mostraron ajustes significativos y valores relativamente altos de $R^{2}$ y devianza explicada. Las proyecciones de la variabilidad climática regional mostraron diferencias sustanciales respecto al incremento monotónico de las salidas de TSM de modelos globales. La técnica de re-escalamiento aquí empleada para los mares mexicanos mostró ser útil para integrar la variación histórica con diferentes criterios de forzamiento.
\end{abstract}

Palabras clave: Escenarios de Cambio Climático, patrones climáticos históricos, pronósticos climáticos, clima oceánico, representatividad climática regional

\section{INTRODUCCIÓN}

En las últimas 2 décadas se ha incrementado el esfuerzo de investigación para explicar y predecir las causas, efectos y tendencias futuras del clima global, incorporando a la acumulación de gases de invernadero como factor determinante (IPCC 2014). Dichas predicciones permitirían prever estrategias de planificación de 
actividades productivas que se podrían ver afectadas por el clima y diseñar las medidas preventivas de mitigación y adaptación a los cambios por venir (Meinke et al. 2006, Asrar \& Hurrell 2013). Las proyecciones de los efectos del calentamiento global se basan principalmente en simulaciones de complejos modelos climáticos que son seleccionados por su relativo realismo en términos de reproducir la variabilidad climática observada (e.g., Van Oldenborgh et al. 2005, Lin 2007, Belmadani et al. 2010).

Los modelos de circulación general (MCG) representan insuficientemente la variabilidad regional, debido a la resolución espacial limitada en sus componentes (océano o atmósfera), así como a limitaciones en los procesos físicos que son capaces de resolver (Parrish \& Peterson 1988). Los errores generados en los MGCA se retienen y amplifican al pasar de una resolución a otra cuando se regionaliza, debido a que la incertidumbre crece conforme aumenta el número de cálculos implicados en la mejora de detalle geográfico (Pielke \& Welby 2011). El modelo desarrollado por el Instituto de Investigaciones Meteorológicas de Japón (MRI/JMA), por ejemplo, consiste en un conjunto de algoritmos acoplados que buscan emular el sistema climático de la Tierra con una resolución horizontal de 5,6 $6^{\circ}$ x 5,6 $6^{\circ}$ y 20 niveles en la vertical para la componente atmosférica, mientras que en la oceánica es de $2,8^{\circ}$ x 2,8 $8^{\circ}$ en la horizontal con 17 niveles verticales. Así como el modelo japonés, existen otros modelos de circulación general que han sido considerados por el Panel Intergubernamental de Cambio Climático (IPCC, por sus siglas en inglés) para predecir la respuesta del clima, sugiriendo diferentes 'futuros posibles', también llamados escenarios (IPCC 2014).

Los escenarios son instrumentos de pronóstico que parten de diferentes hipótesis del comportamiento del clima y van desde supuestos pesimistas hasta moderados. Por ejemplo, entre la familia de escenarios A1 se encuentra el $\mathrm{A} 1 \mathrm{~B}$, que considera a un mundo futuro con rápido crecimiento económico, una población mundial que alcanza su máximo hacia mediados del presente siglo y disminuye posteriormente, así como una rápida introducción de tecnologías nuevas y más eficientes (IPCC 2014). Este escenario supone un equilibrio entre las magnitudes de uso de energías fósiles y de otras fuentes (IPCC 2014). Si bien los escenarios son una herramienta útil para anticipar riesgos asociados al clima en sectores tan diversos como el productivo o la salud pública, la propagación de la incertidumbre en los MGCA no permite obtener una representación adecuada de la variabilidad climática regional (Gutiérrez \& Pons 2006, Pielke \& Welby 2011).

En complemento a las proyecciones generadas por los MGCA, el análisis de los patrones en la variabilidad climática permite generar mejores representaciones de la variabilidad regional y poner en un contexto histórico el efecto de cualquier escenario que se desee evaluar. En este sentido, los mares mexicanos se encuentran bajo la influencia de procesos océano-atmósfera, y los registros históricos muestran patrones de cambio con cierta regularidad que se presentan en diferentes escalas espacio-temporales (Mantua \& Hare 2002, Lluch-Cota et al. 2003, Lluch-Belda et al. 2009). El Pacífico, por ejemplo, presenta 2 señales claras en la temperatura superficial, por un lado la señal ecuatorial de los eventos El NiñoOscilación del Sur (ENSO), y por otro, la oscilación decadal del Pacífico norte (PDO), con un gradiente latitudinal en el que la firma del PDO aumenta hacia el polo, mientras que el efecto del ENSO se concentra en latitudes más tropicales (Lluch-Cota et al. 2003). Este gradiente latitudinal también fue observado en el Golfo de California (Lluch-Belda et al. 2009). En el océano Atlántico se presenta una señal de baja frecuencia identificada como la Oscilación Multidecadal del Atlántico (AMO), un modo de variación que al parecer responde a cambios en la circulación termohalina (Enfield et al. 2001) y que imprime su señal a las fluctuaciones de largo plazo en la temperatura superficial del mar en aquella región.

Los patrones de la variabilidad histórica de la TSM no es considerada de manera explícita por los MGCA debido a que se concentran en la solución determinística de las relaciones físicas entre un gran número de variables (Peixoto et al. 1992). Por tal motivo, la búsqueda de alternativas para mejorar la representatividad regional de las proyecciones de la TSM en mares mexicanos puede aportar en la construcción de plataformas sobre la cual los tomadores de decisiones puedan evaluar escenarios de vulnerabilidad y con ello diseñar estrategias de mitigación y adaptación más adecuadas para cada sector (e.g., pesca, agricultura, salud pública, protección civil). De lo anterior que el objetivo del presente trabajo fue construir proyecciones regionales de la temperatura superficial del mar con el fin de generar alternativas para evaluar escenarios de cambio climático en mares mexicanos, bajo el contexto de sus patrones históricos de variabilidad. 


\section{Materiales Y MÉTODOS}

Para cubrir el objetivo del presente estudio se analizaron 3 conjuntos de datos; la temperatura superficial del mar (TSM), la temperatura superficial del aire (TSA) y diferentes índices climáticos. Se obtuvieron las series de TSM a partir de las reconstrucciones extendidas de temperatura superficial del mar (ERSST, por sus siglas en inglés $)^{1}$. La resolución espacial de los ERSST es de $2^{\circ} \times 2^{\circ}$ y la resolución temporal es mensual con una extensión de 156 años (1858-2010). Se trabajó con los promedios anuales de TSM por cuadrante y se calculó el promedio entre cuadrantes cuando la región incluyó a más de uno (Fig. 1).

Para el análisis de la TSM en aguas mexicanas se tomaron cuadrantes (Fig. 1) dentro de la zona económica exclusiva cuyas regiones presentan propiedades oceánico-biológicas particulares. También se utilizaron los promedios anuales de los datos digitalizados de TSA de los estados costeros mexicanos del entonces Instituto Nacional de Ecología (actualmente INECC) ${ }^{2}$. Por último, las series de los índices climáticos se obtuvieron originalmente de diversas fuentes y se encuentran integrados en la página de internet del Centro Interdisciplinario de Ciencias Marinas del Instituto Politécnico Nacional (CICIMAR-IPN) ${ }^{3}$.

\section{ESTRATEgia GENERAL}

En todos los casos se consideró a la TSM como variable de respuesta, mientras que las variables explicativas fueron la TSA y los índices climáticos. La relación entre las variables de respuesta (TSM) y las variables explicativas fue descrita mediante modelos aditivos generalizados (GAM) para cada región marina (Fig. 1). En los GAM, la TSA fue la variable representante del clima regional, mientras que los índices climáticos aportaron la información de los procesos de gran escala. Para identificar las regiones terrestres con patrones de cambio comunes se aplicó un análisis de regresión y clasificación, aplicando el método de Ward como criterio de enlace (Ward 1963) y el coeficiente de disimilitud de Bray-Curtis (1-r) como medida de distancia (Legendre \& Legendre 1998). El corte del gradiente de similitud se realizó al $70 \%$.

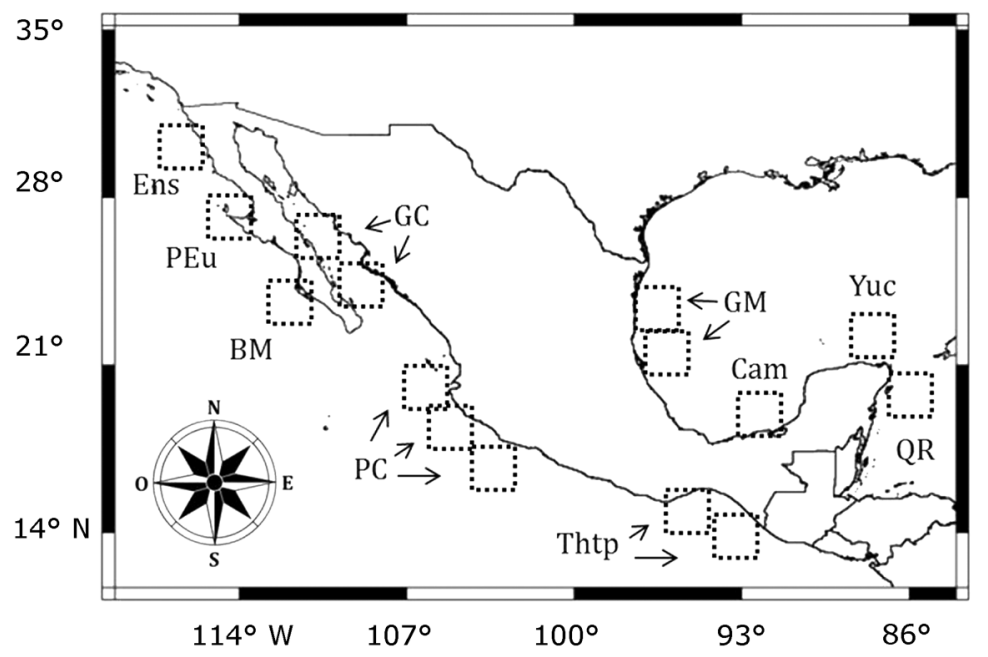

Figura 1. Área de estudio y regiones consideradas: Ensenada (Ens), Punta Eugenia (PEu), Bahía Magdalena (BM), Pacífico Central (PC), Tehuantepec (Thtp), Golfo de México (GM), Campeche (Cam), Yucatán (Yuc) y Quintana Roo (QR) / Considered study areas and regions: Ensenada (Ens), Punta Eugenia (PEu), Bahía Magdalena (BM), Pacífico Central (PC), Tehuantepec (Thtp), Golfo de México (GM), Campeche (Cam), Yucatán (Yuc) and Quintana Roo (QR)

${ }_{1}^{1}<$ http://Iwf.ncdc.noaa.gov/oa/climate/research/sst/sst.php>

${ }^{2}$ INECC. 2013. El cambio climático en México, información por estado y sector. <http://www2.ine.gob.mx/cclimatico/edo_sector/ estados/estados.html>

${ }^{3}<$ www.cicimar.ipn.mx/oacis/Indices_Climaticos.php> 
Para la construcción de cada GAM se tomaron los datos de TSA regional como variable explicativa fija, agregando e intercambiando indicadores climáticos para posteriormente retener en el modelo a aquellos que produjeran el mejor ajuste ( $\mathrm{R}^{2} \mathrm{y}$ devianza explicada). Una vez seleccionado el modelo GAM que mejor se ajustó por región marina, se analizaron las variables explicativas mediante técnicas de series de tiempo, conocidas como descenso cíclico y regresión periódica (Bliss 1958, Bloomfield 1976). Dichos análisis se efectuaron con la ayuda de la función Periods (González-Rodríguez et al. in prep. $)^{4}$, la cual se explica más adelante. Los periodos, amplitudes y fases (PAF) que fueron detectados por la función se utilizaron para extender las series de variables explicativas al año 2080.
Los PAF identificados en las series históricas se utilizaron para obtener valores suavizados de las variables explicativas (TSA + índices climáticos) con el fin de reajustar cada GAM y así dar énfasis a las señales de mediana y baja frecuencia, referentes a la variabilidad decadal y multidecadal. Posteriormente, se realizó la proyección de las series de TSM al año 2080 (Fig. 2). A esta proyección, se le denominó 'Neutral' y sobre ella se aplicaron 3 forzamientos más que consideran un escenario de crecimiento socioeconómico medio-alto (A1B; IPCC 2014) bajo 3 estrategias diferentes que se describen a continuación:

Tabla 1. Principales periodos detectados (línea de trazos) en las series temporales de los índices climáticos y la temperatura superficial del aire por región; BBSS= Baja California, Baja California Sur, Sonora y Sinaloa; NCJMG= Nayarit, Colima, Jalisco, Michoacán y Guerrero; CO=Chiapas y Oaxaca; TVY= Tamaulipas, Veracruz y Tabasco; YQC= Yucatán, Quintana Roo y Campeche. Las líneas punteadas indican los periodos más recurrentes / Main detected periods (depicted dashed lines) of temporal series on climatic indexes and air's superficial temperature by region: (i) BBSS= Baja California, Baja California Sur, Sonora and Sinaloa; (ii) NCJMG= Nayarit, Colima, Jalisco, Michoacán and Guerrero; (iii) $\mathrm{CO}=$ Chiapas and Oaxaca; (iv) TVY= Tamaulipas, Veracruz and Tabasco; (v) YQC= Yucatán, Quintana Roo and Campeche

\begin{tabular}{|c|c|c|c|c|c|c|c|c|c|c|c|c|c|}
\hline \multirow{2}{*}{$\begin{array}{c}\begin{array}{c}\text { Índice } \\
\text { climático }\end{array} \\
\text { PDO }\end{array}$} & \multicolumn{11}{|c|}{ Periodos detectados } & \multirow{2}{*}{$\begin{array}{c}\mathrm{R}^{2} \\
0,422\end{array}$} & \multirow{2}{*}{$\begin{array}{c}P \\
1,25 * \mathrm{e}-08\end{array}$} \\
\hline & & 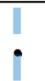 & & & 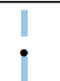 & $\Pi$ & & & & 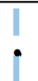 & & & \\
\hline $\mathrm{AMO}$ & - & " & & - & • & I & & & & , & - & 0,664 & $<2,2 * \mathrm{e}-16$ \\
\hline PCI & & 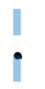 & & - & ! & ! & & & & $!$ & & 0,380 & $1,33 * \mathrm{e}-09$ \\
\hline NOI & & I & & & I & i & & - & & I & • & 0,295 & $<0,003$ \\
\hline BBSS & & 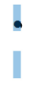 & & • & $\begin{array}{l}\text { I } \\
\text { I }\end{array}$ & 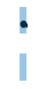 & • & & • & $\begin{array}{l}\text { I } \\
\text { I }\end{array}$ & - & 0,786 & $<2,2 * \mathrm{e}-16$ \\
\hline NCJMG & & $\bullet$ & • & & I & 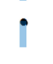 & & & & I & - & 0,392 & $1,67 * \mathrm{e}-06$ \\
\hline $\mathrm{CO}$ & & i & • & & ॥ & I & & & & I & & 0,514 & $2,49 * \mathrm{e}-10$ \\
\hline TVT & & • & • & & ! & ! & & & & ! & & 0,520 & $1,41 * \mathrm{e}-10$ \\
\hline YQC & & • & & • & i & • & • & & • & i & & 0,877 & $<2,2^{*} \mathrm{e}-16$ \\
\hline & 67 & 50 & 40 & 33 & 25 & 19 & 15 & 13 & 11 & 9 & 7 & & \\
\hline & & & & & & & & & & & & & \\
\hline
\end{tabular}

${ }^{4}$ González-Rodríguez E, H Villalobos, VM Gómez-Munoz \& D Lluch-Belda. In prep. A function for extracting periodicities in regular time series. Centro de Investigación Científica y de Educación Superior de Ensenada (CICESE - Unidad La Paz) y Centro Interdisciplinario de Ciencias Marinas (CICIMAR-IPN). 


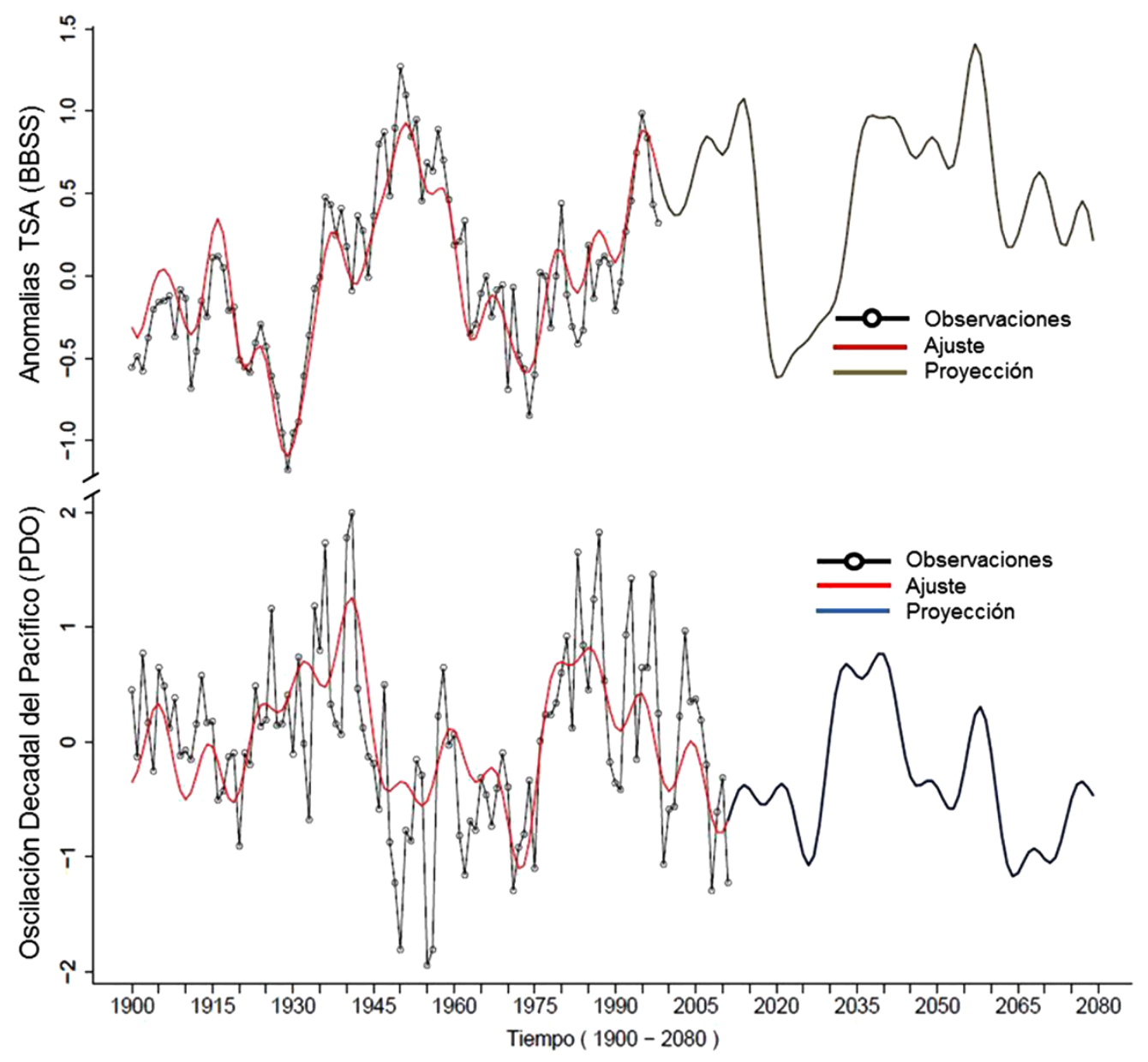

Figura 2. Ajuste (1900-2000) y proyección (al 2080) de los componentes armónicos de las anomalías de temperatura superficial del aire (TSA) en la región BBSS comprendida por los estados de Baja California, Baja California Sur, Sonora y Sinaloa (arriba). Abajo se muestra el ajuste y proyección de la Oscilación Decadal del Pacífico, PDO (1900-2080) / Model fitting (from 1900 to 2000) and projection (leading to 2080) of the harmonic components of the air's surface air temperature anomalies (TSA) in the region BBSS (integrated by Baja California, Baja California Sur, Sonora and Sinaloa). Below, Model fitting and projection of the Pacific Decadal Oscillation, PDO (from 1900 leading to 2080)

1. Proyección Neutral: Predicción de la TSM como salida del modelo GAM. Por medio de la ecuación de regresión periódica (ecuación 2) se extendieron los patrones de cambio (e.g., periodos, amplitudes y fases) detectados en el registro histórico de la TSA y los índices climáticos (ver Fig. 2). Los periodos considerados para cada serie de tiempo se resumen en la Tabla 1. Con la porción de las series extendidas se corrieron las predicciones de TSM mediante modelos GAM, usando las variables explicativas correspondientes a cada región marina según se muestra en la Tabla 2.

2. Japonés: Esta proyección se basa en la proyección neutral más la pendiente $(0,02)$ de las salidas de TSM del modelo japonés (bajo el escenario A1B) para cada región marina. El modelo japonés (CCSR/NIES) es un modelo de circulación general de la atmósfera que busca emular el sistema climático de la Tierra (Resolución atmosférica $=$ $5,6^{\circ}$ x 5,6 $6^{\circ}$, con 20 niveles en la vertical; Resolución oceánica $=2,8^{\circ} \times 2,8^{\circ}$ con 17 niveles verticales).

3. PEACC: Predicciones de TSM provenientes de la proyección neutral más la tasa de cambio lineal $(0,031)$ de las proyecciones de TSA de los Programas Estatales de Acción Contra el Cambio Climático (INECC 2013)². La pendiente se sumó sólo después de correr el GAM. Dado que la magnitud y proporción de los cambios de temperatura son diferentes en el mar y en el aire, se agregó como factor de corrección la relación lineal que tuvo con su región marina aledaña $(0,17)$. Las proyecciones de los programas estatales incluyen el efecto del escenario A1B (INECC 2013) ${ }^{2}$. El escenario A1B supone un crecimiento 
Tabla 2. Síntesis del segundo ajuste en los modelos GAM por región marina, presentando sus valores de coeficiente de determinación $\left(\mathbf{R}^{2}\right)$ y devianza explicada (\%) / Synthesis of the second GAM model fitting by marine region, showing the coefficient of determination values $\left(R^{2}\right)$ and the deviance explained (\%)

\begin{tabular}{lccc}
\hline \multicolumn{1}{c}{ Región Marina } & Fórmula & $\mathrm{R}^{2}$ & $\begin{array}{c}\text { Devianza } \\
\text { explicada (\%) }\end{array}$ \\
\hline Ensenada & $\sim$ BBSS + PDO + PCI & 0,62 & 74 \\
Punta Eugenia & $\sim$ BBSS + PDO + PCI & 0,64 & 76 \\
Bahía Magdalena & $\sim$ BBSS + PDO + NOI & 0,70 & 85 \\
Golfo de California & $\sim$ BBSS + PDO + PCI & 0,62 & 73 \\
Pacífico Central & $\sim$ CO + PCI + TSI + NOI & 0,45 & 55 \\
Tehuantepec & $\sim$ CO + AMO + PDO & 0,52 & 64 \\
Golfo de México & $\sim$ TVT + AMO & 0,54 & 61 \\
Campeche & $\sim$ YQC + AMO & 0,51 & 55 \\
Yucatán & $\sim$ YQC + AMO & 0,60 & 65 \\
Quintana Roo & $\sim$ YQC + AMO & 0,62 & 68 \\
\hline
\end{tabular}

socioeconómico con equilibrio entre el uso de energías fósiles y de otras fuentes (IPCC 2014).

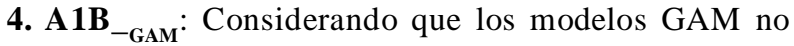
representan relaciones fijas entre variables, sino que son sensibles al entorno de los valores de entrada, se evaluó el desempeño del GAM. Este es un criterio de forzamiento similar al anterior (PEACC). La diferencia consistió en que la pendiente se sumó antes de correr la predicción de TSM.

\section{Modelos Aditivos Generalizados (GAM)}

En un modelo aditivo generalizado el predictor lineal es explicado parcialmente por una suma de funciones suavizantes de las variables predictivas (Wood 2006). Adicionalmente, estos modelos permiten incorporar la familia de distribución del error más adecuada según la variable de respuesta. En este caso se empleó la familia Gaussian e identity como función de enlace debido a que los datos fueron de tipo continuo. En los GAM no existe una ecuación que represente la relación entre la variable respuesta y las explicativas de manera constante, sino que tal ecuación varía según el entorno de valores respuesta de interés gracias a las funciones suavizantes, de ahí que la ventaja de esta aproximación analítica es su gran flexibilidad para modelar relaciones complejas entre las variables de respuesta y sus predictores (Hastie \& Tibshirani 1990). La representación matemática de los modelos aditivos generalizados aplicados a la temperatura superficial del mar (TSM) se ejemplifica a continuación:

$\mathrm{TSM}=\mathrm{X}_{i} * \theta+f_{1}\left(\mathrm{TSA}_{i}\right)+f_{2}\left(\right.$ Índice Climático1 $\left.{ }_{i}\right)$

donde:

$\mathrm{X}_{\mathrm{i}}=$ es una fila de la matriz del modelo para sus componentes estrictamente paramétricos

$\theta=$ es el vector de parámetros correspondientes

$f_{j}=$ representa las funciones suavizantes de las variables explicativas (covariables)

\section{Patrones de Cambio históricos}

Para el análisis de los patrones de cambio en los registros históricos de variables climáticas se emplearon las técnicas de descenso cíclico (Bloomfield 1976) y regresión periódica (Bliss 1958). Tales técnicas fueron combinadas y complementadas con pruebas parciales de $\mathrm{F}$ que evalúan secuencialmente el aporte de cada periodo al 
ajuste global. González-Rodríguez et al. (in prep.) ${ }^{4}$ propusieron y sistematizaron este análisis acoplado de series de tiempo en una función llamada Periods, escrita tanto en código de Matlab como de R. En este estudio se utilizó la versión escrita en R (R Core Team 2014).

El primer paso en el proceso es aislar el componente cíclico de la serie de tiempo ajustando una regresión lineal para determinar la tendencia y removerla de la serie original. El primer periodo a evaluar se toma del tercer valor de la serie en las unidades originales mientras que el valor final depende de la longitud de la serie. Se procede a buscar la amplitud y fase correspondientes mediante la ecuación de regresión periódica:

$$
Y_{t}=a_{0}+a_{1} \cdot \cos (\omega t)+b_{1} \cdot \sin (\omega t)
$$

donde $\omega=2 * p^{-1}$ es la frecuencia angular y $p$ es el periodo.

Los parámetros $a_{1}$ y $b_{1}$ son desconocidos y pueden ser estimados por medio de una regresión múltiple. Se relacionan a la amplitud y el ángulo de la fase por medio de las siguientes ecuaciones:

$$
\begin{gathered}
A=\sqrt{a_{1}^{2}+b_{1}^{2}} \\
\theta=\arctan \left(b_{1} / a_{1}\right)
\end{gathered}
$$

El desfase gráfico del origen puede ser calculado como (ec. 5):

$$
F=\frac{p \cdot \theta}{2 \pi}
$$

La propuesta de González-Rodríguez et al. (in prep. $)^{4}$ implica la evaluación secuencial de cada valor en un conjunto dado de periodos, estimando los parámetros del modelo $\left(a_{1} y_{1} b_{1}\right)$ mientras se prueba qué modelo es el que mejor ajusta a los datos.

El periodo óptimo (op) se selecciona evaluando un criterio gráfico y analítico. Dicho criterio es llamado el Recíproco Máximo de la Suma de Cuadrados Residuales (MRRSS). Analíticamente, el objetivo consiste en ajustar los parámetros de una función del modelo que mejor ajuste a los datos originales con base en la Suma de Cuadrados Residuales (RSS), gráficamente los valores cercanos a cero son amplificados y representan picos que denotan al periodo óptimo.
La representación matemática es como sigue:

$$
M R R S S=\max _{i p \leq p \leq f p}\left(\frac{1}{R S S_{p}}\right)
$$

donde ip es el periodo inicial, $f p$ el periodo final y RSS es definido por:

$$
R S S_{p}=\sum_{t=1}^{n}\left(Y_{t}-Y_{p}\right)^{2}
$$

$\mathrm{Y}_{t}$ es la serie de tiempo sin tendencia y $\mathrm{Y}_{p}$ es la serie ajustada correspondiente al periodo $p$.

Una vez que el periodo óptimo ha sido encontrado (junto con $A$ y $\theta$ correspondientes), la serie ajustada $\mathrm{Y}_{p}$ se convierte en $\mathrm{Y}_{o p}$ y es sustraída de $\mathrm{Y}_{t}$ produciendo una serie de residuales $\left(Z_{\text {res }}\right)$. La regresión periódica será entonces aplicada subsecuentemente a la serie $Z_{\text {res }}$ con el fin de identificar el periodo óptimo con el criterio MRRSS para cada uno de los armónicos $i$ en la serie de tiempo. Este proceso de descenso cíclico (Bloomfield 1976) se repite hasta que el último armónico significativo $m$ es detectado.

Para determinar si la adición de un nuevo periodo es estadísticamente significativa, se estima de acuerdo con una prueba de grados de libertad (Sokal \& Rohlf 1995):

$$
F=\frac{\left(R_{2}^{2}-R_{1}^{2}\right) /\left(k_{2}-k_{1}\right)}{\left(1-R_{2}^{2}\right) /\left(n-k_{2}-1\right)}
$$

donde:

$\mathrm{R}_{2}$ es el coeficiente de determinación del modelo que incluye hasta el harmónico $i$

$\mathrm{R}_{1}$ es el coeficiente de determinación del modelo que incluye hasta el armónico $i-1$

$\mathrm{K}_{2}$ son el número de parámetros en el modelo que incluye hasta el armónico $i$

$\mathrm{K}_{1}$ son el número de parámetros en el modelo incluyendo hasta el armónico $i$ - 1

$\mathrm{N}$ es la longitud de la serie de tiempo

Conforme esta prueba de significancia se realiza, las series $\mathrm{Y}_{o p i}$ se suman simultáneamente para formar la serie ajustada $\mathrm{Y}_{\text {cum }}$. Es por eso que Periods permite evaluar paso a paso el ajuste global sobre la serie original y ello habla del efecto combinado de los patrones identificados $(\mathrm{PAF}=p, A$ y $\theta)$ y no únicamente de la cantidad de energía concentrada en cada periodo (\% de varianza total explicada), como suele suceder en técnicas más 
convencionales como el análisis espectral o el análisis de ondículas (Bloomfield 2000).

\section{Resultados}

De acuerdo con los resultados del árbol de regresión y clasificación (no se muestra), los promedios anuales de la temperatura superficial del aire (TSA) en los Estados de las costas mexicanas, mostraron rasgos comunes de variabilidad, formando 5 grupos: 1) Baja California, Baja California Sur, Sonora y Sinaloa (BBSS); 2) Nayarit, Colima, Jalisco, Michoacán y Guerrero (NCJMG); 3) Chiapas y Oaxaca (CO); 4) Tamaulipas, Veracruz y Tabasco (TVT); 5) Yucatán, Quintana Roo y Campeche (YQC).

Todos los modelos ajustados mediante la función Periods fueron significativos $(P<0,005)$, presentando coeficientes de determinación entre 0,87 y 0,29 (Tabla 1). El modelo con el mayor ajuste sugiere que la TSA de la región YQC puede explicarse en poco más del $87 \%$ de la variabilidad total $\left(P<2,2 * \mathrm{e}^{-16}\right)$ utilizando los periodos de 50, 33, 25 19, 15, 11 y 9 años (Tabla 1 ).

Los siguientes ajustes altos contuvieron combinaciones de los mismos periodos identificados en la región YQC ( 50, 25, 19 y 9 años), explicando más del 50\% de la variabilidad total observada; BBSS (78\%), AMO (66\%), TVT y CO $(>50 \%)$. El periodo de $\sim 50$ años fue detectado en todas las series de TSA e índices climáticos excepto en el índice de oscilación del Pacífico Norte (NOI), posiblemente debido a la corta extensión de la serie, 1948presente (Tabla 1). Los siguientes ciclos más importantes según su persistencia entre series fueron los de 19, 25 y 9 años (Tabla 1). En cuanto a los modelos GAM, todos mostraron coeficientes de determinación relativamente altos $\left(\mathrm{R}^{2}\right.$ entre 0,45 y 0,69$)$ y arrojaron porcentajes de devianza explicada entre 54,7 y 84,5\% (Tabla 2). El mejor ajuste se obtuvo para Bahía Magdalena (Fig. 4c) ya que en esa región intervienen eventos climáticos de mayor frecuencia, mismos que estuvieron bien representados por el NOI, cuyos componentes periódicos fueron de 12, 17 y 5 años (Tabla 1).

Una vez identificados los patrones de cambio en cada variable independiente (índices climáticos y TSA), se utilizaron los periodos, amplitudes y fases para proyectar cada serie al futuro. A manera de ejemplo se detallan las proyecciones obtenidas para el PDO y la TSA de la región BBSS (Fig. 2).

En los registros históricos de anomalías de TSA en la porción noroeste de México (BBSS) se presentaron 2 periodos de enfriamiento, de 1916 a 1930 y de 1955 a 1975 y se esperaría un escenario térmico similar hacia los años 2010-2025. Por su parte, los periodos de calentamiento de 1930 a 1955 y de 1975 a 2010, sugieren que hacia los años 2020-2055, se podrían presentar condiciones de calentamiento similares. La variación común de la TSA en esta región (BBSS), fue explicada en un $78 \%$ por el efecto combinado de los periodos de 50, 33, 19, 15, 11 y 7 años (Tabla 1). Los mismos periodos también fueron detectados en índices climáticos de gran escala como el PDO y el PCI (Tabla 1).

Los cambios en la oscilación decadal del Pacífico (19002010) han presentado 2 fases de calentamiento; de 1910 a 1940 y de 1970 a 1998 (Fig. 2). Según la proyección del indicador, en el Pacífico Norte se esperaría un nuevo periodo de incremento entre el año 2025 y el 2040. Los procesos de enfriamiento también se han presentado de manera recurrente, de acuerdo con las fluctuaciones históricas de este índice, el primer periodo se presentó del año 1940 a 1970 y el segundo de 1990 al 2010. Ya como parte de la proyección se esperaría una nueva fase de enfriamiento hacia los años 2040-2070 (Fig. 2).

En términos generales los modelos GAM fueron buenos predictores utilizando variables representadas por sus patrones de cambio de mediana y baja frecuencia. Como ejemplos de la importancia de la variabilidad de larga duración se puede mencionar a los modelos de las regiones del Golfo de México (norte), Quintana Roo, Campeche y Yucatán (Fig. 5), en los que fue posible explicar la variabilidad de la TSM en 50\% o más, mediante un solo indicador climático (AMO), cuyas principales escalas de variación van de los 9 a los 67 años (Tabla 1).

Los modelos correspondientes a las regiones marinas más norteñas del lado Pacífico presentaron los mejores ajustes con más del $70 \%$ de devianza explicada y valores de $\mathrm{R}^{2}$ superiores a 0,6. En tales modelos, además de la TSA, se adicionó el PDO para representar al componente oceánico del sistema climático y el PCI para el atmosférico, aunque para la región de Bahía Magdalena el NOI representó mejor los cambios en la atmósfera (Tabla 2) debido a que contiene cambios más frecuentes. Los parámetros de ajuste fueron inferiores para el resto de los modelos pero se mantuvieron por encima de 0,45 y $54 \%$ para el coeficiente de determinación y la devianza explicada respectivamente. Los ajustes deficientes de las regiones Pacífico central y Tehuantepec parecen ser un reflejo de las señales climáticas que se combinan en la zona, haciéndose evidente por las variables explicativas seleccionadas en los modelos finales de aquellas regiones (e.g., AMO; Tabla 2). 
De manera gruesa se puede observar que los criterios de forzamiento con los que fueron construidos los escenarios térmicos en todas las regiones marinas, insinúan condiciones más cálidas de las que se podrían esperar por las variaciones históricas. El comportamiento histórico, proyecciones y escenarios térmicos de la TSM en Ensenada (Figs. 3 y 4) y Punta Eugenia (Fig. 4) fueron similares; presentaron una fase de calentamiento entre 1910 y 1940, seguida de un proceso de enfriamiento que se extendió hasta 1975 pero que fue interrumpido por un fuerte y súbito calentamiento que indujo el evento El Niño de 1958-59.

Otro cambio rápido entre 1975 y 77 se observó en las series de TSM de ambas regiones marinas, lo que coincide con el cambio de régimen más nítido y mejor descrito en el Pacífico Norte hasta la fecha, tanto en variables físicas como biológicas (Ebbesmeyer et al. 1990). El proceso de largo plazo asociado al cambio de régimen implicó un calentamiento que se extendió de 1975 a 1998. Además,
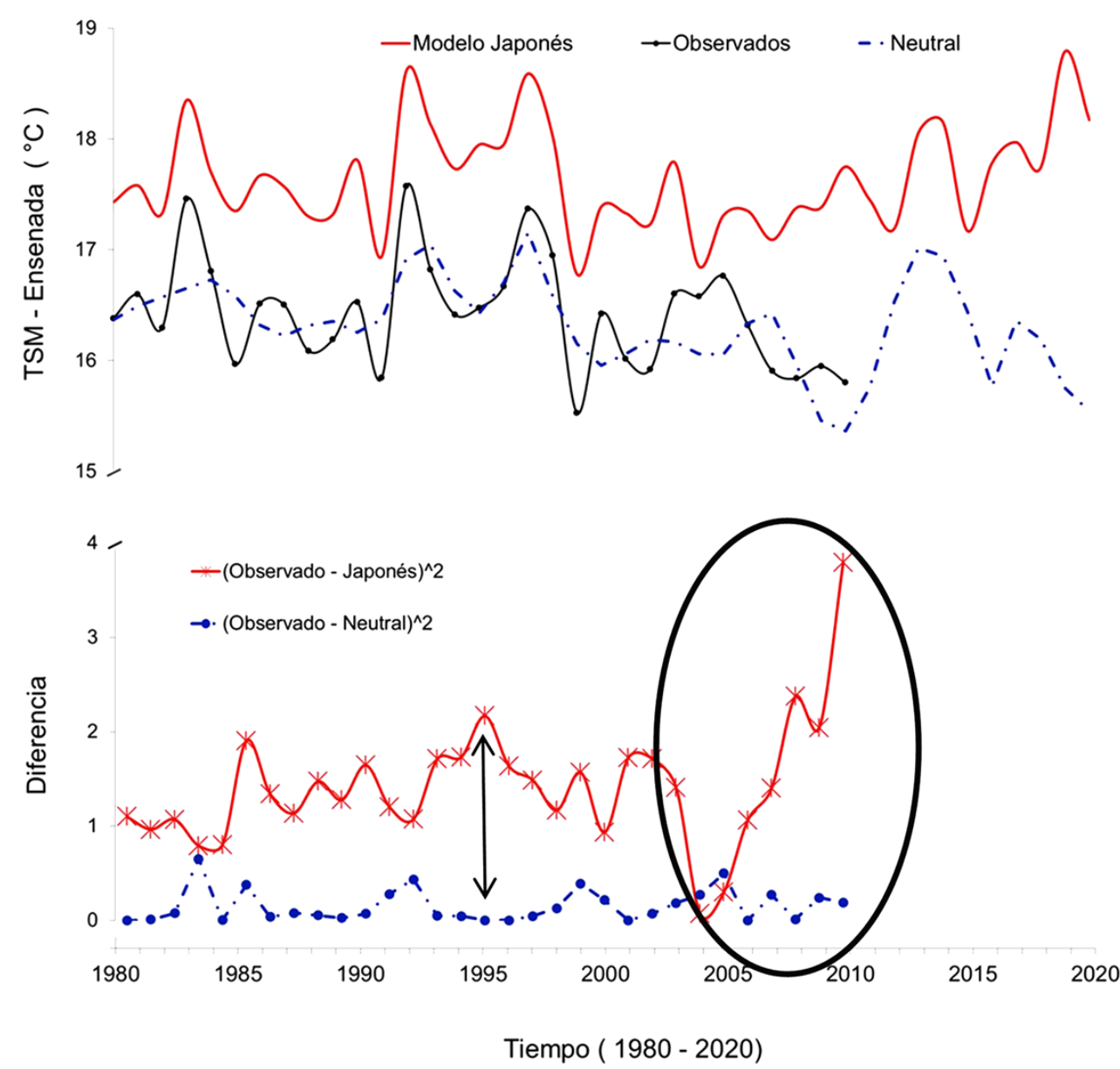

Figura 3. Comparación de la temperatura superficial del mar frente a Ensenada entre las salidas del modelo japonés, los valores observados (ERSST) y las salidas del modelo neutral que fue construido con base en los patrones históricos / Historical comparison of the temperature patterns at sea's surface in Ensenada amongst the Japanese model output, the observed ERSST values, and the neutral model outputs 

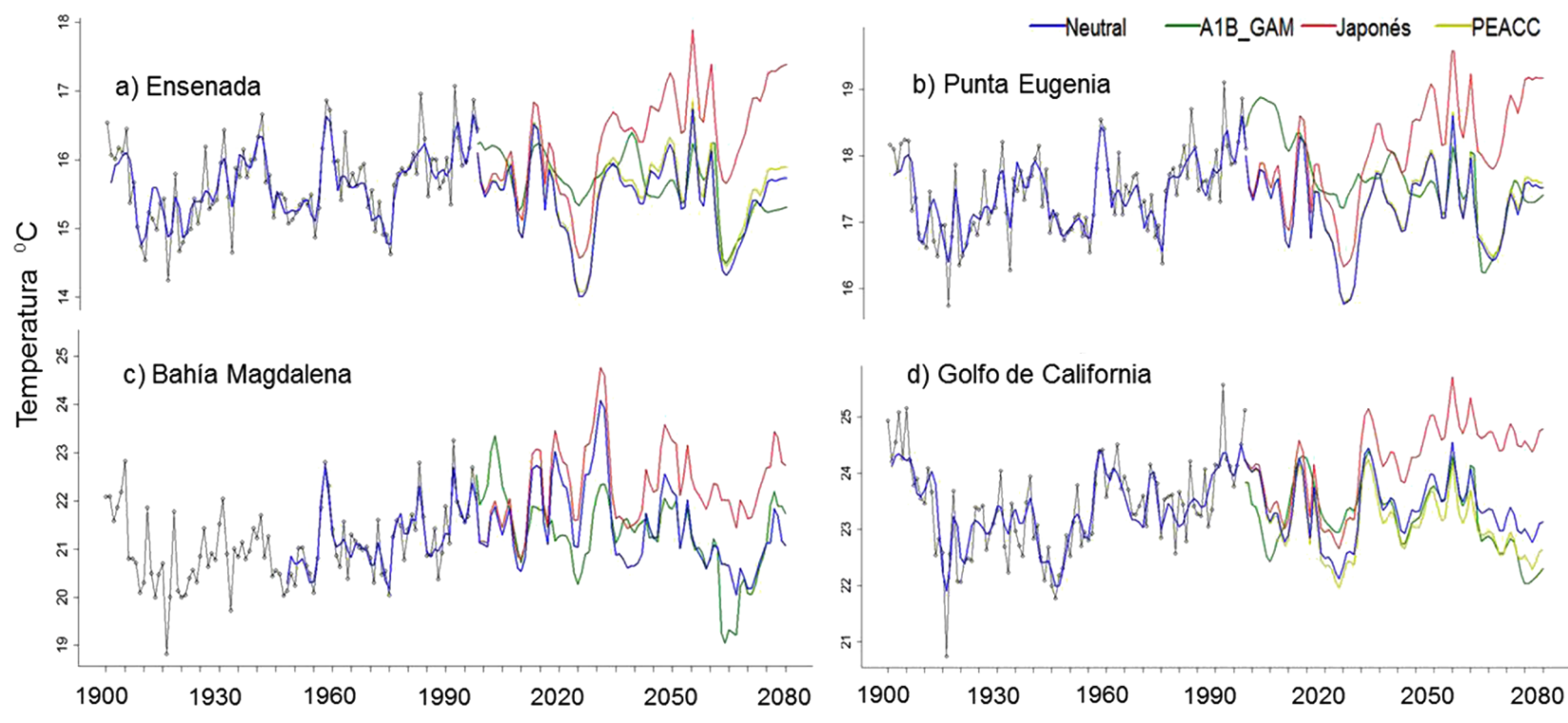

Tiempo (años)

Figura 4. Ajuste (>1900-1998) y proyecciones (1998-2080) de la temperatura superficial del mar en la porción norteña del Pacífico mexicano: a) Ensenada, b) Punta Eugenia, c) Bahía Magdalena y d) Golfo de California / Model fitting (>1900-1998) and projections (1998-2080) of the sea surface's temperature for the north part of the Mexican Pacific: a) Ensenada, b) Punta Eugenia, c) Bahia Magdalena and d) Gulf of California

de la mezcla de registros históricos y resultados de las proyecciones, se puede inferir que un segundo proceso de enfriamiento interdecadal se extienda del año 1999 al 2025. Por último, considerando ya solamente valores proyectados, los resultados sugieren el incremento promedio de la TSM entre los años 2025 y 2055, seguidos por un descenso relativamente rápido los 10 años posteriores, retornando finalmente a una fase de calentamiento (aproximadamente del orden de $1,5^{\circ} \mathrm{C}$ ) hacia el año 2080 (Figs. 3 y 4). Por su parte, los 3 escenarios térmicos que resultaron de cada forzamiento, presentaron el mismo comportamiento fluctuante que la proyección neutral pero con diferencias en las magnitudes de los valores puntuales, por ejemplo; el forzamiento del modelo japonés (línea roja) sugiere un escenario más cálido en poco más de $1{ }^{\circ} \mathrm{C}$ para el periodo $2025-2055$ respecto al resto de los criterios de forzamiento (Figs. 4a y 4b). Las fluctuaciones de largo plazo en las regiones de Bahía Magdalena y el Golfo de California son similares a las 2 localidades más norteñas del estudio (Ensenada y Punta Eugenia), pero parecen presentarse en magnitudes y frecuencia distinta seguramente debido a que reciben de manera más directa la influencia de los cambios interanuales provocados por eventos El Niño (Figs. 4c y 4d).
Los cambios históricos de la TSM en las regiones marinas del Golfo y Caribe de México muestran fases de calentamiento y enfriamiento interdecadal muy similares, seguramente en respuesta a que comparten el forzamiento de la oscilación multidecadal del Atlántico (Fig. 5). Los patrones históricos y la proyección neutral (en azul) de la TSM en conjunto, mostraron 3 periodos de calentamiento interdecadal, 1910-1940, 1975-2010 y 2040-2080, así como 2 de enfriamiento, 1940-1975 y 2010-2040 (Fig. 5). El rango de variación histórica de la TSM en las 4 regiones del lado Atlántico fue de $1,5^{\circ} \mathrm{C}$, pero aumentaría entre $2,5 \mathrm{y}$ $3^{\circ} \mathrm{C}$ si en el futuro se presenta el escenario que sugiere el forzamiento del modelo japonés (línea roja). El forzamiento denominado PEACC afectó de manera similar $\left(\sim 1,5^{\circ} \mathrm{C}\right)$ el rango de variación de la TSM entre las regiones marinas de Yucatán y Campeche (Figs. 5b y 5c), aunque su efecto resultó apenas perceptible respecto a la proyección neutral de las regiones del Golfo de México y Quintana Roo (Figs. 5a y 5d).

Las fluctuaciones históricas en el Pacífico central y el Golfo de Tehuantepec muestran un rango de variación entre 2 y $1,5^{\circ} \mathrm{C}$ respecto a los promedios del periodo 1900 1998. Ese rango de variación se mantiene en los escenarios térmicos futuros, excepto el del modelo japonés que extiende el rango del calentamiento en 0,8 y 1,8 


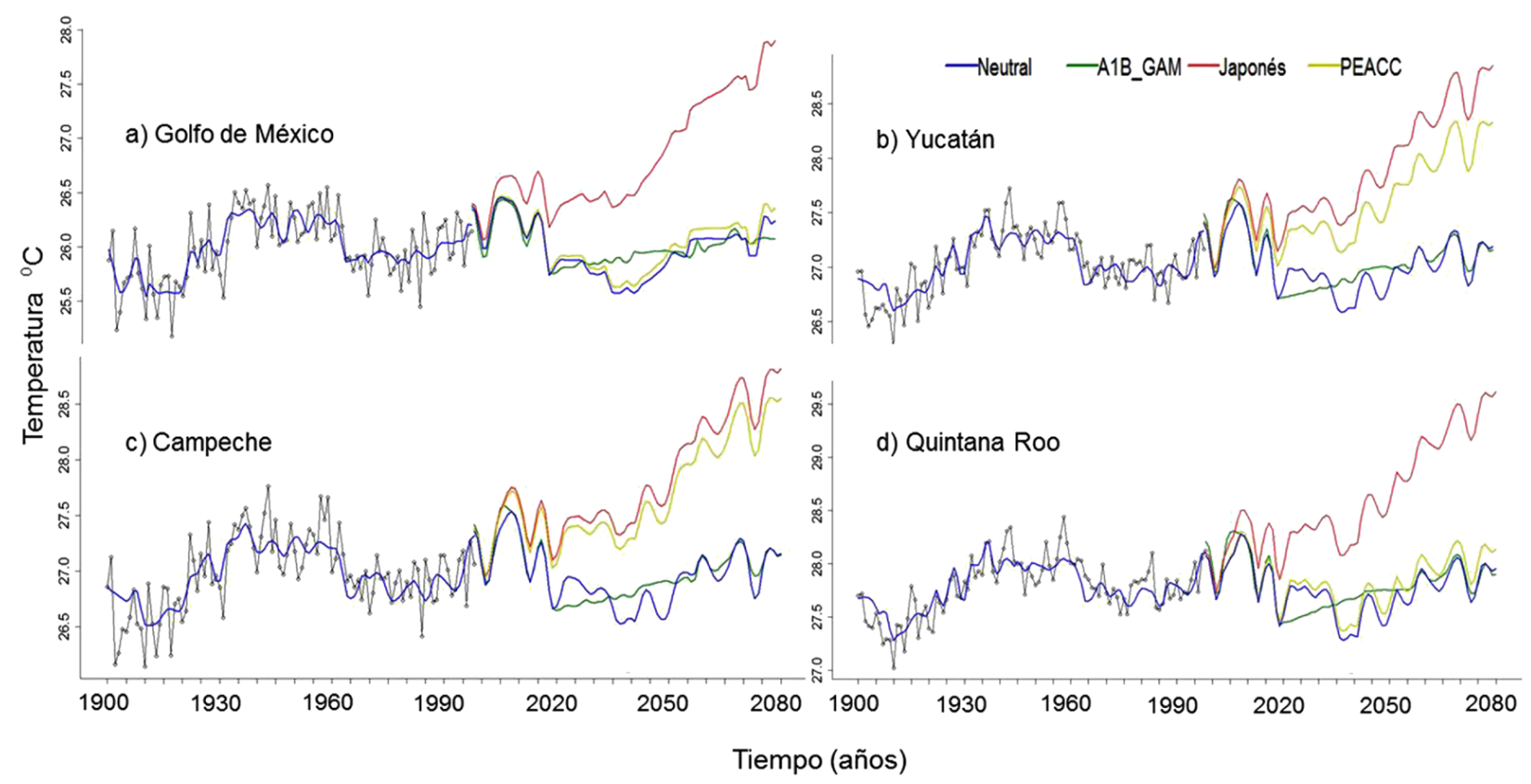

Figura 5. Ajuste (>1900-1998) y proyecciones (1998-2080) de la temperatura superficial del mar: a) Golfo de México, b) Yucatán, c) Campeche y d) Quintana Roo / Model fitting (>1900-1998) and projections (1998-2080) of the sea surface's temperature: a) Golfo de México, b) Yucatán, c) Campeche and d) Quintana Roo

respectivamente (Fig. 6). La sucesión de periodos calentamiento/enfriamiento en el registro histórico de la región se presenta con mayores diferencias en su duración respecto a las regiones de Ensenada y Punta Eugenia, donde fueron más claras. Tal comportamiento en el Pacífico central responde a la influencia tropical que recibe de la corriente mexicana, lo que provoca un mayor peso de los procesos interanuales que de los interdecadales (Figs. 6a y b).

\section{Discusión}

En este estudio se construyeron proyecciones de temperatura superficial del mar (TSM) en coherencia con las fluctuaciones históricas regionales (Fig. 1). Se tomó como base la variabilidad en el pasado y se infiere acerca de lo que podría suceder en el futuro (Figs. 3-6). La proyección Neutral (e.g., Fig. 3) fue elaborada solamente con la información de las series históricas y fue utilizada como contexto de la variación natural en los escenarios térmicos futuros construidos con 3 criterios de forzamiento (A1B_GAM, Japonés y PEACC). Estos forzamientos buscan dar cuenta del impacto antropogénico sobre el clima (e.g., Fig. 4), brindando la posibilidad de evaluarlos en el futuro para calibrar nuestras herramientas de pronóstico. Por otro lado, la presencia de fluctuaciones periódicas en la naturaleza nos permite extender en el tiempo algunas variables de interés, pero para hacerlo con cierta confianza es necesario explicar en algún grado los mecanismos a través de los cuales se manifiestan e interactúan los diferentes patrones de variación.

De acuerdo con la tendencia de la investigación en geofísica, es evidente el interés por estudiar los diferentes fenómenos con el propósito de descubrir patrones de variabilidad, primero con el propósito de explicarlos, y en segundo lugar, con el objetivo de tener capacidades de predicción (Burroughs 2003, Mörner 2010). Sobre este punto, Keller (1999) menciona que es más factible predecir señales de baja frecuencia (largo periodo) que señales de alta frecuencia (corto periodo). Pero probablemente depende de la identificación del mecanismo que produce la señal, y su predictibilidad; como sería el caso para los mecanismos externos como las mareas sol-lunares (Munk \& Bills 2007, Trenary \& Han 2012) o mecanismos de variabilidad interna como El Niño-Oscilación del Sur (Tziperman et al. 1994, IPCC 2007). De lo anterior, que los patrones de cambio detectados en la TSM se podrían atribuir a componentes de variabilidad semiperiódica, de corto y largo plazo (Lluch-Belda et al. 2002, Lluch-Cota et al. 2003). Las variaciones diurna y estacional del ambiente en la mayoría de los ambientes son una manifestación del 


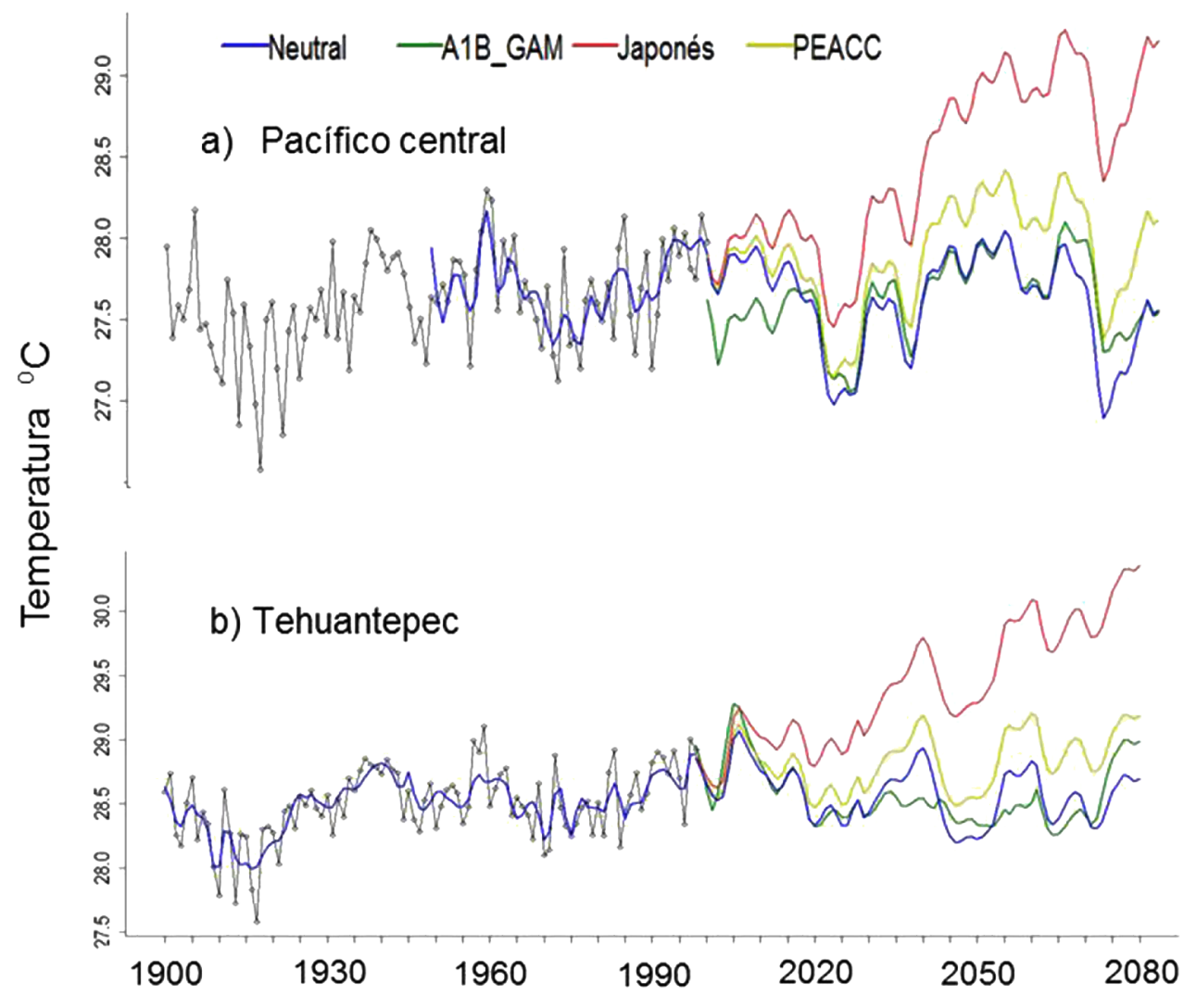

Figura 6. Ajuste (>1900-1998) y proyecciones (1998-2080) de la temperatura superficial del mar: a) Pacífico Central y b) Tehuantepec / Model fitting (>1900-1998) and projections (1998-2080) of the sea surface's temperature: a) Central Pacific and b) Tehuantepec

componente cíclico (de alta frecuencia) como consecuencia de movimientos regulares y predecibles de nuestro planeta (e.g., movimiento de rotación y traslación; Bliss 1958). Pero la identificación y proyección de señales de baja frecuencia (e.g., multidecadales) es oscurecida porque contienen una mayor cantidad de ruido, eventos de corto plazo, eventos súbitos de gran magnitud (e.g., erupciones volcánicas) y la extensión de los registros históricos suelen ser cortos (Lluch-Belda et al. 2002, 2003). A pesar de estas dificultades, algunas señales de baja frecuencia también se asocian a señales geofísicas con cambios cuasi regulares, como la velocidad de rotación de la Tierra o la actividad solar (Mörner 2013, Clilverd et al. 2006).

El comportamiento cíclico se ha reportado de forma recurrente en múltiples fenómenos y eventos naturales (Burroughs 2003). Entre ellos se pueden citar los pulsos rítmicos en grandes corrientes marinas (Morner 2010), los ciclos de formación y ruptura del supercontinente (Nance et al. 1988), las órbitas planetarias (Scafetta 2010), los ciclos de Milankovitch (Haigh 2011) y los ciclos solares (Benestad 2006).

La energía proveniente del sol en forma de radiación electromagnética es el factor fundamental del sistema climático de La Tierra (Haigh 2011). Al entrar al planeta la irradiancia solar interactúa con factores (e.g., nubes, mareas, estacionalidad) que transforman su señal en otras formas de energía (Benestad 2006), por lo que sus efectos son indirectos. La transformación de la energía radiante del sol podría explicar por qué la variable de irradiancia solar total no apareció como variable explicativa en la mayoría de modelos GAM, excepto en el Pacífico central (Tabla 2). 
El índice de circulación atmosférica del Pacífico (PCI) fue seleccionado como variable predictiva en los modelos construidos para Ensenada, Punta Eugenia, Golfo de California y Pacífico Central (Tabla 2; Figs. 4a-d). De acuerdo con sus autores, el PCI representa una forma de medir la transferencia de energía de la atmósfera al océano y eventualmente a la productividad oceánica (King et al. 1998). Si bien este indicador atmosférico describe los cambios en intensidad y dirección predominante de los vientos, su construcción numérica (integral de las anomalías negativas de circulación meridional) se enfoca en el proceso de baja frecuencia (50 60 años), de tal forma que es considerado un forzante de largo plazo (King et al. 1998).

Ahora bien, no todas las regiones responden igual a los forzantes de largo plazo, por lo que se consideró al índice de la oscilación del Pacífico norte (NOI) en la construcción de los modelos predictivos para Bahía Magdalena y el Pacífico Central (Figs. 4c y 6). El NOI es un índice relacionado con las celdas de circulación atmosférica (Hadley-Walker) que determinan la intensidad y los componentes de vientos predominantes (Schwing et al. 2002). De acuerdo con Schwing y colaboradores (2002), los valores negativos del NOI en escalas interanuales y decenales se han asociado con el aumento de temperatura en la superficie del océano, la disminución de vientos favorables para la generación de surgencias y un decremento en la biomasa del zooplancton, al menos frente al sur de California. Además, se ha reportado que el NOI presenta un periodo de aproximadamente 14 años, similar al de 15 años en el que se alternan vientos zonales y meridionales en el Atlántico (Klyashtorin \& Lyubushin 2007), por lo que parece representar un proceso similar en el Pacífico, aunque no necesariamente sincrónico.

Además del NOI y el PCI-invernal, el índice de la oscilación decadal del Pacífico (PDO) tuvo un efecto fundamental en los modelos construidos para las regiones de Ensenada (Figs. 3 y 4a), Punta Eugenia (Fig. 4b), Bahía Magdalena (Fig. 4c), Golfo de California (Fig. 4d) y el Golfo de Tehuantepec (Fig. 6b). Asimismo, el potencial predictivo del PDO es coherente con el hecho de que su patrón asociado de anomalías de TSM y presión al nivel del mar, acompañan la evolución temporal de otras anomalías climáticas del Pacífico y regiones terrestres aledañas, entre ellas la precipitación en Norte América y las anomalías de temperatura en el noreste de Asia (Mantua et al. 1997, Mantua \& Hare 2002, Dettinger et al. 1998, Cayan et al. 1998). Al respecto, Schneider \& Cornuelle (2005) sugieren que la variación paralela de procesos oceánicos y atmosféricos en el Pacífico puede deberse a que el PDO actúa como forzante de la atmósfera o bien, a que un forzamiento común actúa sobre el PDO y las anomalías atmosféricas que le acompañan.

En forma similar al PDO, tanto en la señal que representa como en su amplia influencia geográfica, el AMO (oscilación del Atlántico) es un importante modo de variación multidecadal de la temperatura superficial del mar. El AMO ha presentado fases alternadas de calentamiento y enfriamiento que impactan amplias porciones del Hemisferio Norte (Enfield et al. 2001), por lo que tiene un peso importante en la predicción del clima regional (Knigth et al. 2006; Tabla 2). El poder predictivo del AMO en los modelos GAM se encuentra relacionado con su influencia de largo plazo sobre las regiones de Quintana Roo (Fig. 5d), Yucatán (Fig. 5b), Campeche (Fig. 5c) y el Golfo de México (Fig. 5a). La señal del AMO se extiende incluso hacia el Pacífico a través de una teleconexión que atraviesa la región más estrecha del macizo continental mexicano, explicando así porqué fue seleccionada en el modelo de Tehuantepec (Tabla 2; Fig. 6b). Si bien la ciclicidad dominante del AMO es de baja frecuencia (65 80 años), también le fueron detectados patrones de cambio decadales (Tabla 1), pero su relación con procesos de mediana ( $\sim 10$ años) y alta frecuencia $(<5$ años) aún no ha sido resuelta del todo (Gray et al. 2004).

Es fundamental tener presente que las predicciones de TSM necesariamente tienen asociado algún grado de incertidumbre, tanto por la sensibilidad del sistema climático ante cambios en sus factores determinantes (e.g., naturales o antropogénicos), como a la tasa cambiante de absorción de calor del océano (Stott \& Kettleborough 2002). Según AchutaRao et al. 2006, los cambios en la capacidad del océano para absorber calor son una fuente de incertidumbre en las predicciones de modelos acoplados (atmósfera-océano), que se vuelve particularmente relevante en escalas decadales o mayores. En general, la importancia de la variabilidad interna se incrementa conforme la escala espacial y temporal se reduce (Hawkins \& Sutton 2009). La incertidumbre total en los MCG se compone, además de la variabilidad interna, de la incertidumbre generada por el modelo y la asociada al escenario (Räisänen 2001, Hawkins \& Sutton 2009).

La importancia relativa de las 3 fuentes de incertidumbre cambia significativamente con la región, la extensión del pronóstico y la escala del promediado temporal que se aplique (Räisänen 2001). En horizontes temporales de una o dos décadas, las fuentes principales de incertidumbre (en escala regional) provienen del 
modelo y de la variabilidad interna, mientras que en escalas multidecadales, el mayor peso relativo de incertidumbre surge del escenario. De ahí que los registros históricos de temperatura han sido utilizados para ayudar a reducir el rango de incertidumbre que acarrean las predicciones bajo escenarios de calentamiento (Stott \& Kettleborough 2002, Hawkins \& Sutton 2009).

En síntesis, los patrones históricos de cambio de diferentes procesos climáticos de macro escala, mostraron utilidad predictiva sobre la TSM de los mares mexicanos, indicando que a la variabilidad climática regional subyacen patrones detectables que los asocian con procesos de variabilidad interna a escalas mayores, bridando la posibilidad de evaluar diversos escenarios climáticos dentro de contextos más adecuados. Es por ello que las fluctuaciones históricas de la TSM, como variable indicadora del ambiente físico y un conjunto de atributos biológicos relacionados, son un referente que permite generar nociones respecto a lo que podría suceder en los ecosistemas de los mares mexicanos en el futuro. Finalmente, al incorporar la variabilidad histórica de la TSM y evaluar el escenario A1B destaca que deben tomarse en cuenta las fuentes de incertidumbre, así como periodos multianuales de descenso de la temperatura y periodos de relativa estabilidad, aun cuando en el largo plazo se prevea algún escenario de calentamiento.

\section{Agradecimientos}

Este artículo está dedicado a la Memoria del Dr. Daniel Lluch-Belda (1942-2014), un ser humano entrañable que supo transformar su trabajo en ejemplo y en sustanciales aportes a la investigación pesquera nacional e internacional. Las ideas centrales detrás de la presente propuesta se cimientan en una parte de su fructífera labor. ¡Un gran abrazo Doc!

Agradecemos a los sistemas de becas COFAA y EDI del Instituto Politécnico Nacional, así como al CONACYT por la beca de posgrado (Becario No. 219142).

\section{LITERATURA CITADA}

AchutaRao KM, BD Santer, PJ Gleckler, KE Taylor, DW Pierce, TP Barnett \& TML Wigley. 2006. Variability of ocean heat uptake: Reconciling observations and models. Journal of Geophysical Research 111(C05019): 1-20.

Asrar GR \& JW Hurrell. 2013. Climate science for serving society: Research, modeling and prediction priorities, 484 pp. Springer, New York.

Bliss CI. 1958. Periodic regression in biology and climatology. Bulletin of the Connecticut Agricultural Experimental Station New Haven 615: 1-55.
Bloomfield P. 1976. Fourier analysis of time series: An introduction, $258 \mathrm{pp}$. John Wiley \& Sons, New York.

Bloomfield P. 2000. Fourier analysis of time series: An introduction, $288 \mathrm{pp}$. John Wiley \& Sons, New York.

Burroughs WJ. 2003. Weather cycles, 317 pp. Cambridge University Press, New York.

Cayan DR, MD Dettinger, HF Diaz \& NE Graham. 1998. Decadal variability of precipitation over western North America. Journal of Climate 11: 3148-3166.

Clilverd MA, E Clarke, T Ulich, H Rishbeth \& MJ Jarvis. 2006. Predicting solar cycle 24 and beyond. Space Weather 4: S09005 <doi:10.1029/2005SW000207>

Dettinger MD, DR Cayan, HF Diaz \& DM Meko. 1998. North-south precipitation patterns in western North America on interannual-to-decadal timescales. Journal of Climate 11(12): 3095-3111.

Enfield DB, AM Mestas-Nunez \& PJ Trimble. 2001. The Atlantic multidecadal oscillation and it's relation to rainfall and river flows in the continental U.S. Geophysical Research Letters 28: 2077-2080.

Gray ST, LJ Graumlich, JL Betancourt \& GT Pederson. 2004. A tree ring based reconstruction of the Atlantic multidecadal oscillation since 1567 A.D. Geophysical Research Letters 31(12): L12205 <doi:10.1029/ 2004GL019932>

Gutiérrez JM \& MA Pons. 2006. Modelización numérica del cambio climático: bases científicas, incertidumbres y proyecciones para la Península Ibérica. Revista de Cuaternario y Geomorfología 20(3-4): 15-28.

Haigh J. 2011. Solar influences of climate, 20 pp. Imperial College, London.

Hastie TJ \& RJ Tibshirani. 1990. Generalized additive models, 329 pp. Chapman \& Hall, London.

Hawkins E \& R Sutton. 2009. The potential to narrow uncertainty in regional climate predictions. Bulletin of the American Meteorological Society 90: 1095-1107.

IPCC. 2014. Emissions scenarios, 570 pp. Cambridge University Press, Cambridge <http://www.ipcc.ch/ ipccreports/sres/emission/index.php?idp=0>

Keller CF. 1999. Climate, modeling, and predictability. Physica D: Nonlinear Phenomena 133(1): 296-308.

King JR, VV Ivanov, V Kurashov, RJ Beamish \& GA McFarlane. 1998. General circulation of the atmosphere over the North Pacific and its relationship to the Aleutian Low. North Pacific Anadromous Fish Commission Document 318: 1-18.

Klyashtorin LB \& AA Lyubushin. 2007. Cyclic climate changes and fish productivity, $223 \mathrm{pp}$. VNIRO, Moscow.

Knigth JR, CK Folland \& AA Scaife. 2006. Climate impacts of the Atlantic multidecadal oscillation. Geophysical Research Letters 33: 1-4. 
Lamb HH. 1972. Climate: Present, past and future, 581 pp. Methuen, London.

Lin JL. 2007. Interdecadal variability of ENSO in 21 IPCC AR4 coupled CGCMs. Geophysical Research Letters 34: L12702 〈doi:10.1029/2006GL028937>

Lluch-Belda D, DB Lluch-Cota \& SE Lluch-Cota. 2002. Is interannual change predictable at the California Current System? Oceanography of the Eastern Pacific II: 74-85.

Lluch-Belda D, P del Monte-Luna \& SE Lluch-Cota. 2009. 20th century variability in the Gulf of California SST. CalCOFI Report 50: 147-154.

Lluch-Cota DB, WS Wooster, SR Hare, D Lluch-Belda \& A Parés-Sierra. 2003. Principal modes and related frequencies of sea surface temperature variability in the Pacific Coast of North America. Journal of Oceanography 59: 477-488.

Mantua NJ \& SR Hare. 2002. The Pacific decadal oscillation. Journal of Oceanography 58: 35-44.

Mantua NJ, SR Hare, Y Zhang, JM Wallace \& RC Francis. 1997. A Pacific decadal climate oscillation with impacts on salmon. Bulletin of the American Meteorological Society 78: 1069-1079.

Meinke H, R Nelso, P Kokic, R Stone, R Selvaraju \& W Baethgen. 2006. Actionable climate knowledge: from analysis to synthesis. Climate Research 33: 101-110.

Misra V, SM DiNapoli \& S Bastola. 2012. Dynamic downscaling of the twentieth-century reanalysis over the southeastern United States. Regional Environmental Change 13(1): 15-23.

Mörner NA. 2010. Solar minima, Earth's rotation and Little Ice Ages in the past and in the future. The North AtlanticEuropean case. Global and Planetary Change 72: 282-293.

Mörner NA. 2013. Solar wind, earth's rotation and changes in terrestrial climate. Physical Review \& Research International 3(2): 117-136.

Munk W \& B Bills. 2007. Tides and the climate: some speculations. Journal of Physical Oceanography 37: 135-147.

Nance RD, TR Worsley \& JB Moody. 1988. The supercontinent cycle. Scientific American 259: 72-79.
Parrish JT \& F Peterson. 1988. Wind directions predicted from global circulation models and wind directions determined from eolian sandstones of the western United States-A comparison. Sedimentary Geology 56: 261-282.

Pielke RA \& RL Wilby. 2011. Regional climate downscaling. What's the point? Eos, Transactions American Geophysical Union 93(5): 52-53.

R Core Team. 2014. R: A language and environment for statistical computing. R Foundation for Statistical Computing, Vienna. <http://www.R-project.org/>

Räisänen J. 2001. $\mathrm{CO}_{2}$-induced climate change in CMIP2 experiments: Quantification of agreement and role of internal variability. Journal of Climate 14: 2088-2104.

Scafetta N. 2010. Empirical evidence for a celestial origin of the climate oscillations and its implications. Journal of Atmospheric and Solar-Terrestrial Physics 72: 951-970.

Schneider N \& BD Cornuelle. 2005. The forcing of the Pacific Decadal Oscillation. Journal of Climate 18: 4355-4373.

Schwing FB, T Murphree \& PM Green. 2002. The Northern Oscillation Index (NOI): ANew Climate Index for the Northeast Pacific. Progress in Oceanography 53(4): 115-139.

Sokal RR \& FJ Rohlf. 1995. Biometry, 887 pp. W.H. Freeman \& Company, New York.

Stott PA \& JA Kettleborough. 2002. Origins and estimates of uncertainty in predictions of twenty-first century temperature rise. Nature 416: 723-726.

Trenary LL \& W Han. 2012. Intraseasonal-to-interannual variability of South Indian Ocean sea level and thermocline: Remote versus local forcing. Journal of Physical Oceanography 42(4): 602-627.

Tziperman E, L Stone, MA Cane \& H Jarosh. 1994. El Niño chaos: overlapping of resonances between the seasonal cycle and the Pacific ocean-atmosphere oscillator. Science 264: 72-74.

Van Oldenborgh GJ, S Philip \& M Collins. 2005. El Niño in a changing climate: a multi-model study. Ocean Science Discussions 2: 267-298.

Wood SN. 2006. Generalized additive models: An introduction with R, 392 pp. Chapman and Hall / CRC, Boca Raton. 